\title{
QoS Control Method in MMT and Its Implementation
}

\author{
Changkyu Lee*, Sunghei Kim**, Shingak Kang** \\ *Dept. of engineering, University of science and technology, Daejeon, Korea (Republic of) \\ **Electronics and telecommunications research institute, Daejeon, Korea (Republic of)
}

echkyu@etri.re.kr, shkim@etri.re.kr, sgkang@etri.re.kr

\begin{abstract}
MPEG-2 TS had been developed to deliver and to store media data such as audio, video, and non-timed data. And it has been popularly used by various industries and applications. As different type of networks has been converging, there is a need for a new technology which is more familiar with packet network and more suitable for emerging new types of media and service. In order to support the objectives, ISO/IEC JTC1/SC29/WG11 (a.k.a., MPEG) has been developing a standard called MPEG media transport (MMT). In this paper, we described how MMT can support efficient media delivery and also we showed its prototype implementation.
\end{abstract}

Keywords - MPEG media transport (MMT), QoS control method, Implementation

\section{INTRODUCTION}

MPEG-2 TS had been designed to deliver and to store media data such as audio, video, and non-timed data and it has been popularly used by various industries and applications (e.g. digital broadcasting, bluray disk). As different type of networks such as broadcasting network and mobile network has been converging, there is a need for a new technology which is more familiar with packet network and capable to support emerging new types of media and service such as UHD and $3 \mathrm{D}$ video. In order to achieve the objectives, ISO/IEC JTC1/SC29/WG11 (a.k.a., MPEG) has been developing the standard for MPEG media transport (MMT) as the first part of MPEG-H. In order to support efficient delivery of media data, MMT supports QoS control by transmitting media data with different characteristics according to the client-side information. In this paper, we introduced the QoS control method in MMT and showed its implementation. The remaining part of this paper is organized as follows. In section II, we introduced the MMT in functional area point of view. We explained QoS control method of MMT and showed its implementation in section III. We concluded the paper in section IV.

\section{MPEG MEDIA TRANSPORT AND FUNCTIONAL AREAS}

The objective of MMT is efficient delivery of emerging new types of media data in an adaptive fashion over various types of network [3]. For media transport over various packet networks, MMT is designed to consist of four functional areas as shown in Fig.1.

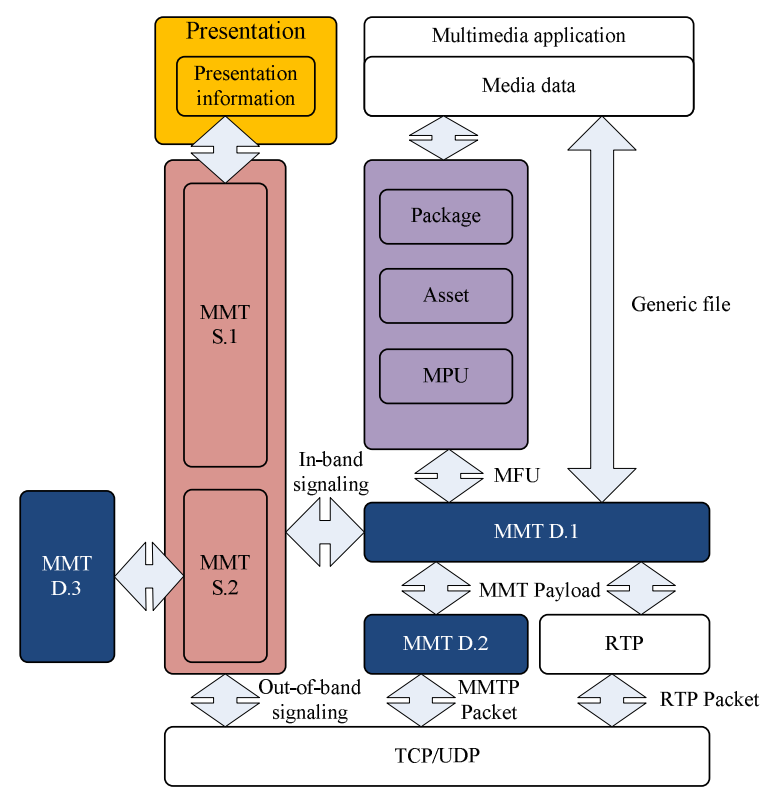

Figure 1. Functional areas of MMT

MPU functional area defines the format for MFU, MPU, Asset, and Package and is divided into three sub-areas which are MMT E.1, MMT E.2, and MMT E.3. Each sub-area generates header and payload for data. MMT E.1, E.2, and E.3 sub-areas generate Package, Asset, and MPU respectively. An MPU (media processing unit) is an entity for processing such as encapsulation, packetization, and presentation in MMT system. As a generic container, an MPU is independent on any specific codec. When an MPU is fragmented for delivery, the fragmentation of media data part is called MFU (media fragment unit). An Asset consists of multiple MPUs and it contains only one type of media data. Asset is the largest data unit for which same presentation information and ADC (Asset delivery characteristics) are applied. Package is not a unit of MMT but is the collection of data including Assets, ADC, and presentation information. An example of Package is shown in Fig.2. 


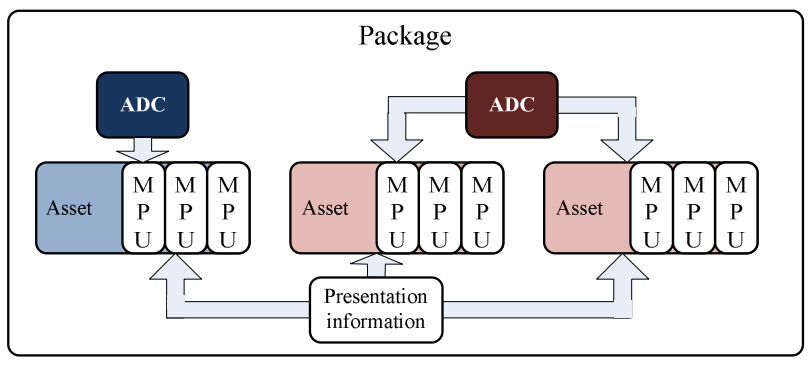

Figure 2. Structure of Package

ADC [7] describes the required network characteristics to deliver each Asset in Package. This indicates that each type of media has the corresponding ADC. For example, the ADC of Asset for audio data is different the ADC of Asset for video data. Presentation information describes the information on the time when each Asset is presented and the position where each Asset is presented. Presentation information is defined by presentation functional area.

Presentation functional area defines a metadata for media presentation. The metadata can include temporal relationship among Assets (presentation time of each media and inter/intramedia synchronization), and spatial relationship among Assets (arrangement of media in one screen/multi-screen). Because presentation information is essential to present media data, presentation information is required to be delivered at service initiation. If presentation information needs to be updated, the new version of presentation information can be sent by MMT sending entity. In order to define description method, ISO/IEC JTC1 SC29/WG11 has been developing 23008-11, MMT composition information [9]. The CI is composed of XML file for temporal information and HTML5 file for spatial information. According to $\mathrm{CI}$, a screen has one view and a view can be composed of one or more Areas where one or more Assets can be presented. If presentation is required to be described for multiple screens, CI can include multiple views. Although CI is developed for MMT, MMT does not restrict use of other presentation description scheme such as MPD defined in ISO/IEC 23009-1.

Signaling functional area provides functionalities to control the media delivery and consumption. Signaling functional area is divided into two sub-areas which are MMT S.1 and MMT S.2. MMT S.1 is designed for media consumption control. For example, $\mathrm{CI}$ is delivered from MMT sending entity to MMT receiving entity through the message belonging to MMT S.1 sub-area. MMT S.2 sub-area is designed for media delivery control. For example, reception quality feedback message and ARQ message belongs to MMT S.2 sub-area. Messages can be delivered through either in-band or out-of-band; MMT considers in-band signaling as a default manner.

Delivery functional area provides functionalities for delivery encapsulated media data from one network entity to another. Delivery functional area is divided into three subareas which are MMT D.1, MMT D.2, and MMT D.3. MMT D.1 sub-area generates MMTP payload which include media data encapsulated in MMT encapsulation functional area; it is Assets in Package. The MMTP payload can be a payload of MMTP packet which MMT D.2 sub-area (MMT protocol, MMTP) generates or be a payload of RTP packet when RTP is used instead of MMTP. This indicates that MMT can be used with RTP to delivery media data over IP network. For crosslayer related operations, MMT defines MMT D.3 sub-area which provides CLI (cross-layer interface) [8] between various network layers and application layer. Through the information gained by CLI, MMT can be aware of the network status and can support appropriate media delivery for each network environment.

\section{QOS CONTROLMETHODS IN MMT}

MMT is designed to have the QoS-related information such as NAM, ADC, and fields in MMTP header. In addition, MMT also has the signaling message related to QoS control. Each information or signaling message can be used for three different types of QoS control method [7].

Per-class QoS control method uses the ADC and MMPT header. When an Asset needs to be transferred, three fields in the header of the corresponding MMTP packets are set based on ADC; "type_of_bitrate" field, "delay_sensitivity field", and "transmission priority" field are set. When the network entity such as "media aware network element (MANE)" receives an MMTP packet with such information, it decides whether the received packet has higher priority than already received packet(s). The result of decision includes packet drop and reordering queue. Per-class QoS control is similar to the operation in router which able to operate based on DSCP value [10].

Per-flow QoS control method relies on the prior resource reservation operation using other protocol such as RSVP [11]. In order to use per-flow QoS control, MMT sending entity needs to reserve resources for each flow according to ADC. During the process, either MMT sending entity or network entity such as MANE needs to allocate flow label for each flow. When MMT sending entity generate MMPT packet, it set "flow label" field appropriately. After the MMTP packet is sent, $\overline{\text { it }}$ will be handled by network entities according to the reservation.

Different from the previous two QoS control methods which try to guarantee the QoS, QoS control for best effort network does not guarantee any quality but it adaptively adjusts the quality according to the status of MMT receiving entity or MMT receiving entity-side network. The QoS control method for best effort network exploits both NAM and end-toend measurement results and it can be classified into two types; server-driven QoS control and client-driven QoS control. In this paper, we focused on how MMT can provide the QoS control method for best effort network.

\section{A. Server-driven QoS Control}

In server-driven QoS control method, MMT sending entity controls the quality of media. For example, MMT sending entity can change the quality of video from HD to SD. For server-driven QoS control, MMT receiving entity should report its status such as end-to-end measurement result or network 
status. The type of report, items to be measured, etc. are configured by MMT sending entity. Fig.3 depicts the procedure of server-driven QoS control for best effort network. MMT sending entity sends an MC message for measurement configuration. The MC message contains configuration for items to be measured, measurement period, type of report, and so on. During the service, MMT receiving entity will report the measurement result by sending RQF message or NAMF message. Based on the report, MMT sending entity needs to determine whether adaption is required. If MMT sending entity decides to change the quality of media, it sends media data with different quality.

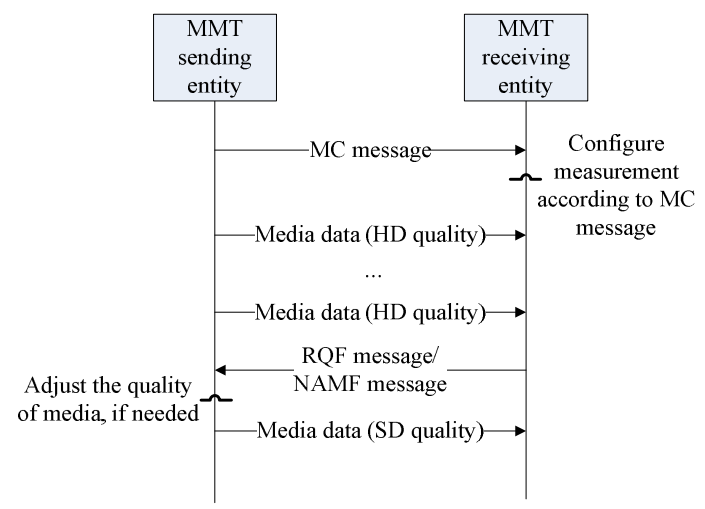

Figure 3. Procedure of server-driven QoS control

\section{B. Client-driven QoS Control}

MMT receiving entity is privileged to choose appropriate Asset in client-driven QoS control. In order to choose suitable Asset, MMT receiving entity needs to get ADCs corresponding to each Asset so MMT sending entity needs to send the information on the mapping between ADCs and Assets to MMT receiving entity at service initiation. MMT receiving entity can conduct client-driven QoS control based on the information. Fig. 4 shows how client-driven QoS control can be conducted. As described above, MMT sending entity sends ADC message containing the mapping information between ADCs and Assets. The ADC message can be described for each Asset. Asset ID information, the ADC corresponding to the Asset and the information on the adaptable Assets will be described for each Asset. After MMT receiving entity receives $\mathrm{ADC}$ message, the MMT receiving entity can request different quality of Asset. In order to conduct client-driven QoS control, MMT receiving entity determines whether adaptation is needed based on ADCs for all adaptable Assets and its network status. When MMT receiving entity decides to change the quality, it sends adaptation request message (AREQ message in Fig.4). The adaptation request message may contain both Asset ID which the MMT receiving entity is receiving, and Asset ID which the MMT receiving entity wants to receive. The request message may contain request for multiple Assets.

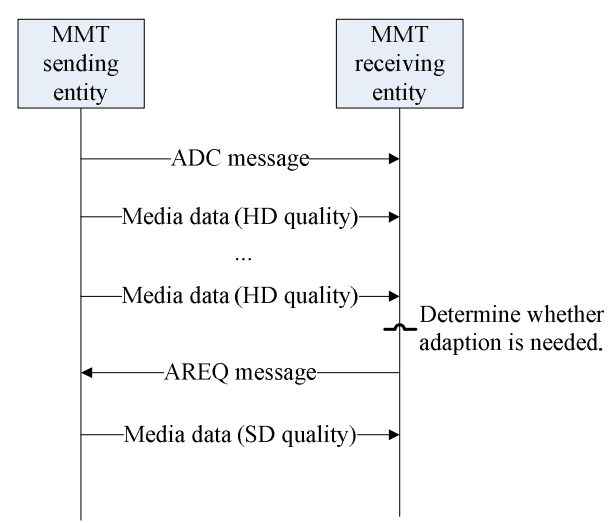

Figure 4. Procedure of client-driven QoS control

Upon receiving the request message, MMT sending entity sends the requested Asset. In client-driven QoS control, MMT sending entity does not determine anything and it just responds to MMT receiving entity.

\section{Implementation of prototype}

The prototype of MMT is developed using GStreamer [12] and $\mathrm{C}++$ on Ubuntu. Because ISO/IEC 23008-1 MMT is still under development in ISO/IEC JTC1 SC29/WG11, we focused on development of QoS control part described in the above. Fig. 4 shows the run-time screen capture of the implemented prototype of MMT sending entity and MMT receiving entity. The MMT sending entity continuously sends media data since it starts; service initiation procedure is not conformed to MMT because we want to focus on QoS control. The MMT receiving entity can send a report message indicating the change of access network; CLI is not implemented so the change of access network is not actually happened. Upon receiving a report message (red box in Fig.5), MMT sending entity checks whether adaptation is needed by comparing the current media quality and the reported access network type. By using the prototype, we can observe server-driven QoS control of MMT works well but more precise determination scheme needs to be developed and implemented.

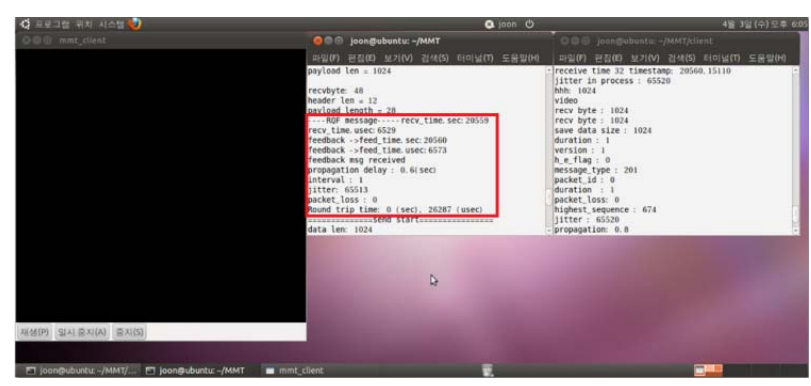

Figure 5. Prototype Implementation

Currently, the prototype supports server-driven QoS control thus it needs be extended to support client-driven QoS control and CLI-related operations. 


\section{CONCLUSIONS}

In this paper, we introduced the standardization status of ISO/IEC 23008-1 MMT. Based on the currently developed document, we implemented the prototype of MMT, especially with respect to server-driven QoS control. With the prototype, we observed server-driven QoS control of MMT works. As future work, the prototype needs to be enhanced to support CLI-related operations and more precise adaptation scheme. Based on the standardization status of MMT, the prototype will be updated.

\section{ACKNOWLEDGMENT}

This research was supported by the ICT Standardization program of MSIP (Ministry of Science, ICT \& Future Planning).

\section{REFERENCES}

[1] Cisco, "Cisco Visual Networking Index: Forecast and Methodology, 2011-2016", May 2012.

[2] ISO/IEC JTC1/SC29/WG11, "N11539, Call for Proposals on MPEG Media Transport (MMT), July 2010.

[3] ISO/IEC JTC1/SC29/WG11, "N11541, Context and Objectives on MPEG Media Transport (MMT), July 2010.

[4] ISO/IEC JTC1/SC29/WG11, "N13462, Requirements on MPEG Media Transport (MMT), April 2013.

[5] ISO/IEC JTC1/SC29/WG11, "N13463, Use Cases for MPEG Media Transport (MMT), April 2013.

[6] ISO/IEC JTC1/SC29/WG11, "N13982, Text of ISO/IEC FDIS 23008-1 MPEG Media Transport", November 2013.

[7] ISO/IEC JTC1/SC29/WG11, "N13983, Study of ISO/IEC 23008-1 DAM 1 Additioanl technologies for MPEG Media Transport", November 2013.

[8] ISO/IEC JTC1/SC29/WG11, "N13987, Text of ISO/IEC 23008-1 DAM 2 Header Compression and Cross Layer Interface", November 2013.

[9] ISO/IEC JTC1/SC29/WG11, "N13989, Text of ISO/IEC DIS 23008-11 MPEG Media Transport CI", November 2013.

[10] IETF, "RFC 2474, Definition of the Differentiated Services Field (DS Field) in the IPv4 and IPv6 Headers", December 1998.

[11] IETF, "RFC 2205, Resource ReSerVation Protocol (RSVP)"., September 1997.

[12] GStreamer, http://gstreamer.freedesktop.org/

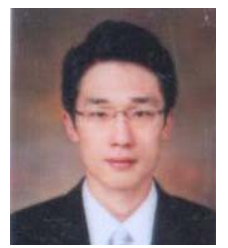

Changkyu Lee received the B.S. in computer science and engineering from Inha University, Korea, in 2008. $\mathrm{He}$ is currently pursuing his M.S. and Ph.D. degree in engineering at University of science and technology, Korea. He is working for ETRI as UST student since 2008 . He is actively participating in standardization in ITU-T study group 11, 13, ISO/IEC JTC 1/SC 6, MPEG. His research interests include peer-to-peer networking, multimedia communications.

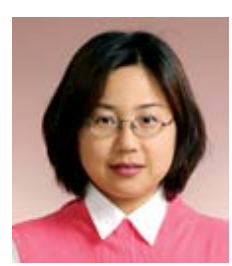

Sunghei Kim received the B.S. and M.S. in computer science from Ehwa University in 1991 and from Chungnam University in 1995, respectively. She is pursuing a Ph.D. degree at Chungnam University. She has been working as a researcher in ETRI since 1991. She is actively participating in standardization in ITUT study group $11,13,16$, ISO/IEC JTC 1/SC 6, MPEG, W3C. Her topic of interests includes, multimedia delivery, peer-to-peer communication, multicasting, future network, and Internet routing.

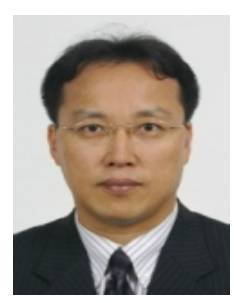

Shin-Gak Kang received the B.S. and M.S. degree in electronics engineering from Chungnam National University, Korea, in 1984 and 1987, respectively and the Ph.D. degree in information communication engineering from Chungnam National University, Korea in 1998. He is working for ETRI since 1984. Currently, he is a Director of media application standard research section. From 2008 he is a professor at the Department of Engineering, University of Science and Technology, Korea. He is actively participating in various international standard bodies as a Vicechairman of ITU-T SG11, Convener of JTC 1/SC 6/WG 7, etc. His research interests include contents networking, multimedia communications, and Future Network. 